\title{
Prostate Multiparametric MRI: Common Pitfalls in Primary Diagnosis and How to Avoid Them
}

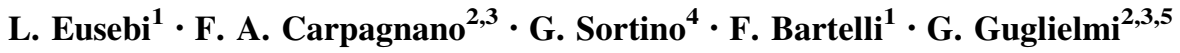

Accepted: 5 January 2021 / Published online: 28 January 2021

(c) The Author(s) 2021

\begin{abstract}
Purpose of Review To provide the radiologist with basic knowledge about normal and abnormal findings in the prostatic mp-MRI, taking a look at the possible diagnostic pitfalls commonly seen in daily clinical practice, allowing him to recognize and consequently avoid them.

Recent Findings Prostate mp-MRI has now become commonly used in most diagnostic imaging centers, as a precise, accurate and above all non-invasive tool, useful in the diagnosis, staging and follow-up of prostate diseases, first of all prostatic carcinoma. For this reason, it is important to take into account the existence of numerous possible anatomic and pathologic processes which can mimick or masquerade as prostate cancer.

Summary Through the combination of anatomical (T2WI) and functional sequences (DWI/ADC and DCE), the mpMRI of the prostate provides all the information necessary for a correct classification of patients with prostate disease, cancer in particular. It is not uncommon, however, for the radiologist to make errors in the interpretation of imaging
\end{abstract}

This article is part of theTopical Collection on Geriatrics.

G. Guglielmi

giuseppe.guglielmi@unifg.it;

http://www.unifg.it

1 Radiology Unit, "Carlo Urbani” Hospital, Jesi, Italy

2 Department of Clinical and Experimental Medicine, Foggia University School of Medicine, Viale L. Pinto 1, 71121 Foggia, Italy

3 Radiology Unit, "Dimiccoli” Hospital, Barletta, Italy

4 Urology Unit, "Carlo Urbani” Hospital, Jesi, Italy

5 Radiology Unit, Hospital "Casa Sollievo Della Sofferenza”, San Giovanni Rotondo, Foggia, Italy due to conditions, pathological or otherwise, that mimic prostate cancer and that, consequently, affect the diagnostic/therapeutic process of patients. The strategy, and what this pictorial review aims at, is to learn to recognize the potential pitfalls of the prostatic mp-MRI and avoid them.

Keywords mp-MRI · Pitfalls · Benign lesions · Prostate cancer

\section{Introduction}

Nowadays, the use of mp-MRI in the diagnosis, staging and therapeutic management of prostate cancer has become very common and widespread. It is well known, in fact, that compared to the canonical diagnostic tools used so far, such as digit rectal exploration (DRE), serum values of specific prostatic antigen (PSA) and prostate trans-rectal biopsy, mp-MRI is able to provide more information for a more accurate diagnostic-therapeutic classification of prostate cancer patients $[1 \bullet, 2]$. All this is possible not only thanks to the intrinsic potential of the imaging method, but also thanks to the optimized and standardized protocols that have been developed over the years. In 2012, in fact, the European Society of Urogenital Radiology developed guidelines, under the name of "Prostate Imaging-Reporting and Data System (PI-RADS)", about the protocols of acquisition and interpretation of MR imaging of prostate cancer [3, 4•]. After a series of revisions to which these guidelines have been submitted, up to the latest version published in 2019 (PI-RADS v2.1), it has been reached to identify milestone sequences of mp-MRI that offer a complete picture of prostatic disease, either anatomically or functionally [5]. In particular, the study of the prostate gland involves the acquisition of anatomical sequences T2- 
weighted imaging (T2WI), in combination with functional sequences of diffusion-weighted imaging (DWI) and dynamic type after intravenous administration of contrast medium (DCE) [4•]. The combined use of functional and anatomic sequences increases the sensitivity and the specificity of mp-MRI in detection of clinically significant prostate cancer. Moreover, it is commonly agreed that among these, there are some sequences which are more or less specific and indicated for the study of the zonal anatomy of the prostate gland: the T2WI sequences seem to be more suitable for the study of the transition zone (TZ) and the DWI sequences for the peripheral zone (PZ) [7•].

Although the existence of a standardized protocol provides considerable support to the radiologist in the prostate imaging interpretation and reporting, the mp-MRI is certainly not free from diagnostic pitfalls, both for the great inter-observers variability in the interpretation between different radiologists, both because many benign conditions or even normal anatomical structures can have similar characteristics to prostatic carcinoma $[7 \bullet, 8]$.

A good knowledge of the anatomy of the prostate gland, as well as of the common behavior and the imaging appearance of the prostatic carcinoma and those conditions that can mimic it, can, therefore, be vital to avoid incurring inconvenient diagnostic pitfalls. In light of the above, the purpose of this review is to provide useful clarifications on potential diagnostic and interpretative pitfalls in the imaging of mp-MR of prostatic cancer and to identify possible strategies to avoid common errors.

\section{Prostate Anatomy}

The starting point for a correct evaluation of the prostate in mp-MRI is represented by a good knowledge of the gland, on the three axial planes, coronal and sagittal, as this allows us to recognize more easily the peri- and intra-glandular anatomical structures, in order to distinguish them from suspicious lesions and avoid running into trivial diagnostic pitfalls.

In a cranial-caudal progression the prostate can be divided into three planes starting from the base, placed immediately below the bladder, the middle third, seat of the verumontanum, and the apex.

Even more important in diagnostic imaging of the prostate, however, is the subdivision of the gland from a histopathological point of view, according to which four distinct areas are recognized: the anterior fibromuscular stroma (AFMS), the transition zone (TZ) surrounding the prostatic urethra in its proximal tract, the central zone (CZ) surrounding the ejaculatory ducts, and the peripheral zone (PZ) where the largest percentage of glandular tissue is concentrated.
Although in T2WI anatomical sequences the distinction between these zones is quite clear and definite, it can happen, especially with changes related to age and $\mathrm{BPH}$, that the volumetric increase of the $\mathrm{TZ}$ causes a distortion of the glandular anatomy by compressing the $\mathrm{CZ}$. In this case the distinction in the two central and transitional portions will no longer be so demarcated and will be recognized, in a more generic way, only one common central gland.

In T2WI morphological imaging it is also possible to appreciate a thin hypointense rhyme, called "prostatic capsule", which is arranged to partially surround the prostate excluding the anterior area and the apex. Knowing how to recognize this anatomical structure, is of vital importance for the evaluation of the extra-capsular extension (ECE) in the staging of prostate cancer. Between TZ and PZ, on the other hand, a similar structure is recognized, which appears as a thin dark line in T2WI sequences, which goes under the name of "pseudo-capsule" or "surgical capsule".

Parallel to the prostate, in postero-lateral location, run the neurovascular bundle which is responsible for vascularization and innervation of the gland, also important in the staging of carcinoma.

\section{Anatomic Structures Mimicking Prostate Cancer at MR Imaging}

\section{Anterior Fibromuscular Stroma}

The anterior fibromuscular stroma (AFMS) is a band of tissue arranged anteriorly to the $\mathrm{TZ}$ of the prostate gland. It consists of smooth and striated muscle fibers, together with connective tissue, arranged to form an anterior compact portion of the prostate, which extends from the base to the apex of the gland, partly on the side surfaces, which shows a thicker thickness in its central part $[1 \bullet, 9,10]$. The AFMS is also susceptible to great individual variability, being able to differ from patient to patient especially from a dimensional point of view. For example, it appears smaller in older patients with a great prostatic volume [11]. Because of its histology, AFMS (Fig. 1) typically shows a homogeneous low intensity signal in T2WI sequences, lenticular morphology [4•], and an equally low signal in the ADC map [9]. Due to the above characteristics and especially in cases of considerable size, this physiological portion of the gland can be interpreted as prostate cancer. What allows us to distinguish it from a suspected malignancy lesion is the possibility to use all the available planes of mp-MRI, including the coronal and sagittal ones, in order to perform a complete and multiplanar evaluation of prostatic anatomy and to confirm the continuity of the AFMS with the surrounding benign tissue. In addition, its behavior in DWI 
sequences and relative ADC map, in which it shows no diffusion restricted phenomena, and after intravenous administration of contrast medium (dynamic DCE sequences), in which it shows no high signal intensity, represents another useful tool for differential diagnosis [12].

Another element that may suggest that the radiologist is faced with a possible diagnostic pitfall is the perfectly central and symmetrical location of the radiological finding [9].

\section{Periprostatic Vein Plexus and Neurovascular Bundle}

The periprostatic neurovascular bundle runs closely in proximity to the prostate surface, in particular the venous
Fig. 1 Hypointense focal area on axial T2WI (a) in the middle-basal anterior gland (arrow), which shows poor restriction on ADC map (b). These described findings are compatible with anterior fibromuscular stroma (AFMS)
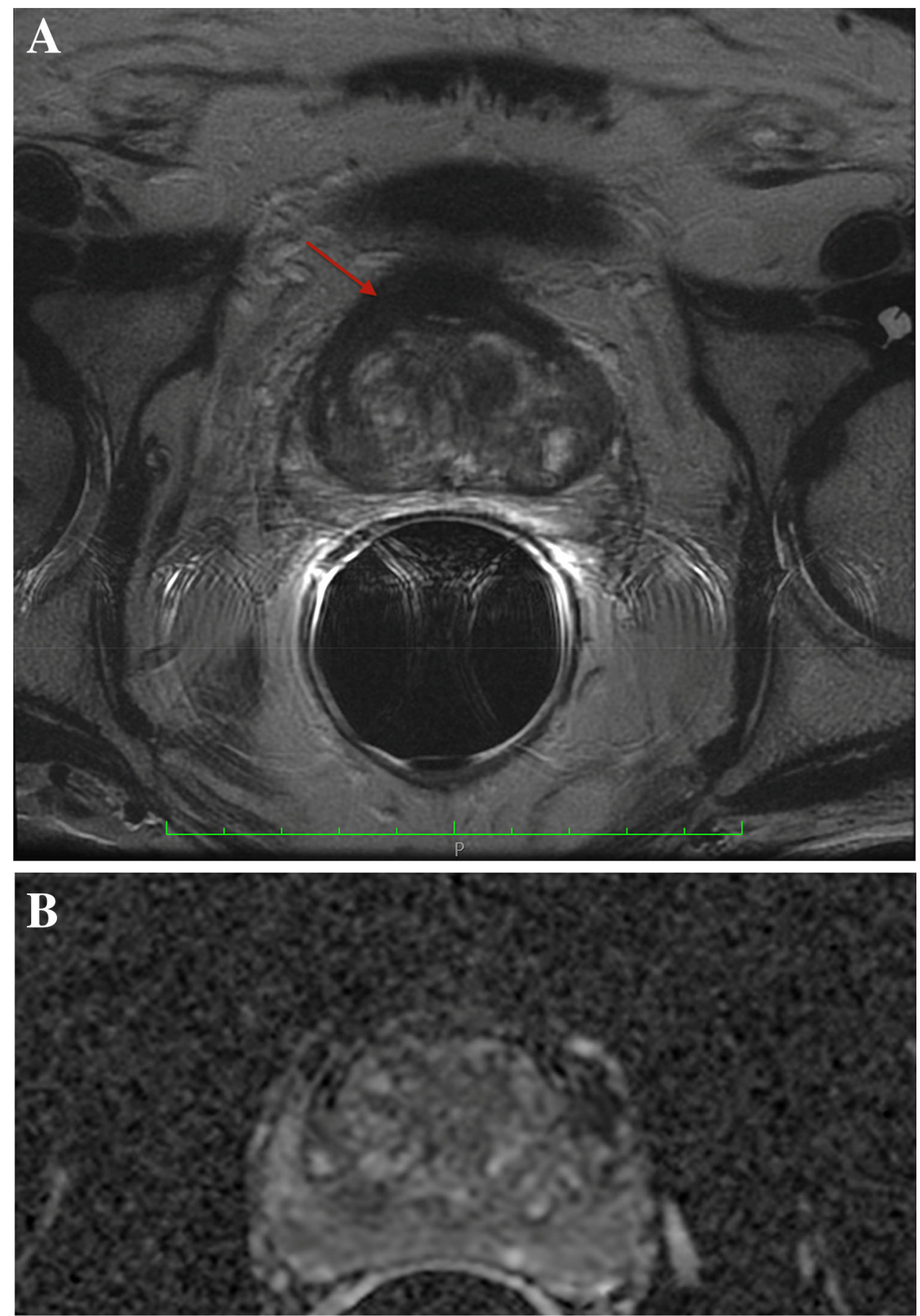
plexus of Santorini, the main responsible for venous drainage of the gland, is located near the antero-surface, while the neurovascular bundle runs along the posterior-lateral surface [13]. The appearance of these anatomical structures can be quite variable, depending on the morphology of the gland, the age of the patient and his clinical conditions; vascular structures, for example, have a higher caliber in young patients with lower prostatic volume, as they appear more pronounced in prostatitis due to their congested appearance $[4 \bullet, 5,6,7 \bullet]$.

Normally the periprostatic veins appear as tubular structures, or roundish on the axial plane, with hyperintense signal in the T2WI sequences; however, due to variations in turbulence and blood flow velocity [9], they may appear as focal round images with low signal intensity, representing a challenge in differential diagnosis with peripheral area prostate cancer (PZ) [6].

Differential diagnosis becomes even more complex due to the appearance and behavior of vascular structures in DWI and dynamic sequences: sometimes the periprostatic venous plexus may show a narrow (hyperintense) diffusion signal in DWI sequences, with a corresponding low signal intensity in the ADC map, and a focal enhancement in DCE sequences [4•].

An aspect such as that described, if it is added to the anatomical localization, can induce to interpret the radiological findings as prostate cancer affecting the PZ and, probably, in stage T3 (tumor extending beyond the prostatic capsule) $[7 \cdot, 8,9,10,11,12,13,14]$.

A practical suggestion for the radiologist facing such a diagnostic pitfall may consist primarily in the multiparametric evaluation of pelvic imaging, especially T2WI anatomical sequences, since vascular structures, unlike tumor, have a linear morphology on the coronal and sagittal planes [9]. In addition, a detailed observation of DWI and dynamic sequences can be useful to differentiate the behavior of venous vessels compared to neoplastic tissue, since the latter shows an earlier and more intense enhancement compared to vascular structures that, On the other hand, they have a slower and moderate intensity enhancement [15].

The neurovascular bundle, similar to the periprostatic venous plexus, may be mistakenly interpreted as a prostatic carcinoma of the PZ, in this case on the postero-lateral side of the gland. Also in this case, a good anatomical knowledge of the periprostatic structures, with a detailed evaluation on the three planes imaging (axial, coronal and sagittal) and the DWI and DCE sequences, can be of help in distinguishing the vascular beam-nervous from a possible prostate carcinoma [4].

\section{Normal Central Zone}

By convention, due to the different histology and embryogenesis, in addition to anatomical reasons, the prostate gland is commonly divided into three distinct parts: the central area (CZ), the transition area (TZ) and the peripheral area (PZ). The central area, a symmetrical conical structure surrounding the ejaculatory ducts from the base to the verumontanum (the point where the ejaculatory ducts flow into the prostatic urethra), is rarely affected by prostate cancer (only $0.5-2.5 \%$ of cases) [9], where it is associated with a higher incidence of extraprostatic extension (ECE), a lower therapeutic response and a higher risk of recurrence [16, 17].

In mp-MRI, the $\mathrm{CZ}$ typically shows a hypointense signal in T2WI anatomical sequences and an equally low signal in the ADC map; these characteristics, although overlapping with those of prostate cancer, do not represent a limit in the differential diagnosis, in particular considering the perfect symmetry and anatomical position of the $\mathrm{CZ}$, as well as its signal homogeneity and uniformity [18].

However, from the age of 35 , the $\mathrm{CZ}$ undergoes a progressive reduction in volume and morpho-structural distortion due to the contextual hypertrophy of the surrounding TZ, thus assuming an asymmetrical appearance. This may be an obstacle in differential diagnosis with prostate cancer, as the hypo-intensity described in the T2WI sequences and the ADC map will appear no longer uniform and symmetrical, but as a focal area of hypo-intensity which can assume on the coronal plane a triangular or teardrop shaped appearance, depending on whether the axial slice level is more cranial or more caudal [15].

In particular, it is very common to find at the base of the prostatic gland median, bilateral and generally symmetrical areas, laterally to the ejaculatory ducts, that appear homogeneously hypointense in T2WI sequences and in the ADC map and have a variable enhancement after administration of contrast medium (Fig. 2). This aspect is defined in the literature as "mustache-sign" and it occurs in cases where there is a homogeneous and bilateral stromal proliferation of the $\mathrm{TZ}$, at the base or at the middle of the gland, which determines a compression of the adjacent CZ, or a real larger adenoma that usually affects the posterior portion of the prostate (mustache-like sign) [4•].

A possible variant or extension of the "mustache-sign" is represented by a focal area of hypo-intensity, visible in T2WI sequences, at the posterior middle third or at the base of the prostatic PZ, which can easily be mistaken for a malignant lesion (Fig. 3). This aspect is called "teardropsign" and is the result of the compression of the central area $(\mathrm{CZ})$ between the $\mathrm{TZ}$ and the $\mathrm{PZ}$ and, therefore, exerts a posterior bulging on the area of verumontanum [4•]. 

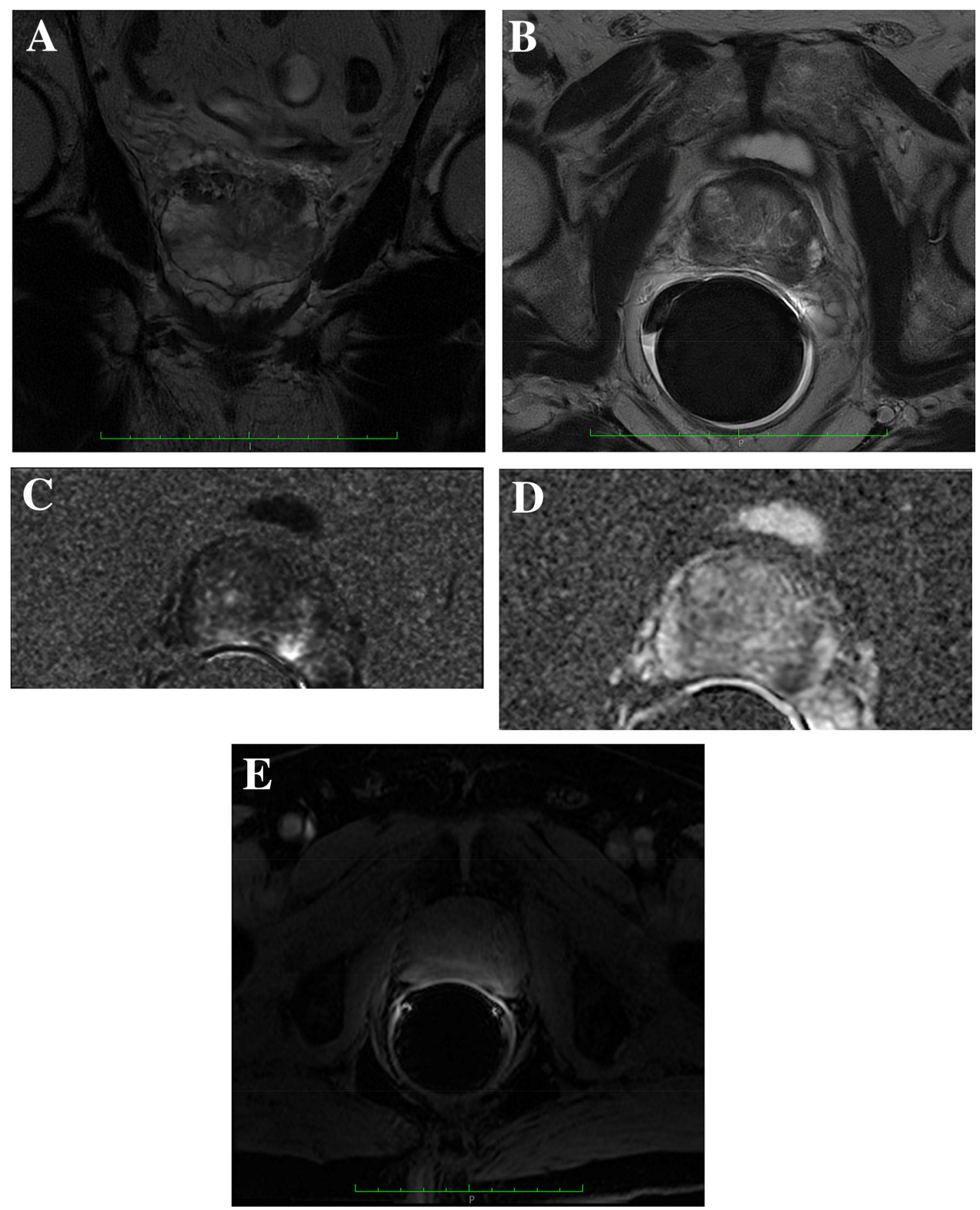

Fig. 2 Two median, symmetric and bilateral areas of low signal intensity on coronal (a) and axial (b) T2WI, showing a restricted diffusion on DWI (c) and ADC map (d) and a diffuse enhancement on DCE (e). This aspect is commonly described as "mustache sign"

The low signal intensity in T2WI morphological sequences is therefore related to the hypertrophic tissue surrounding the ejaculatory ducts before flowing into the urethra and which will be better visible on the coronal plane.

Furthermore, at this point, as well as an accurate anatomical analysis of the gland on three planes of space, it is mandatory to consider the $\mathrm{CZ}$ enhancement curve in DCE sequences: it typically shows a progressive (type 1) or progressive enhancement curve with subsequent plateau (type 2), other than early enhancement and with rapid wash-out (type 3) typical of prostate cancer [19]. 
Fig. 3 Axial (a) and coronal (b) T2WI show a hypointense area at the prostate base, in the peripheral zone (PZ). This aspect is the result of the compression of central zone (CZ) between the transition zone (TZ) and the peripheral zone (PZ), taking a teardrop shape
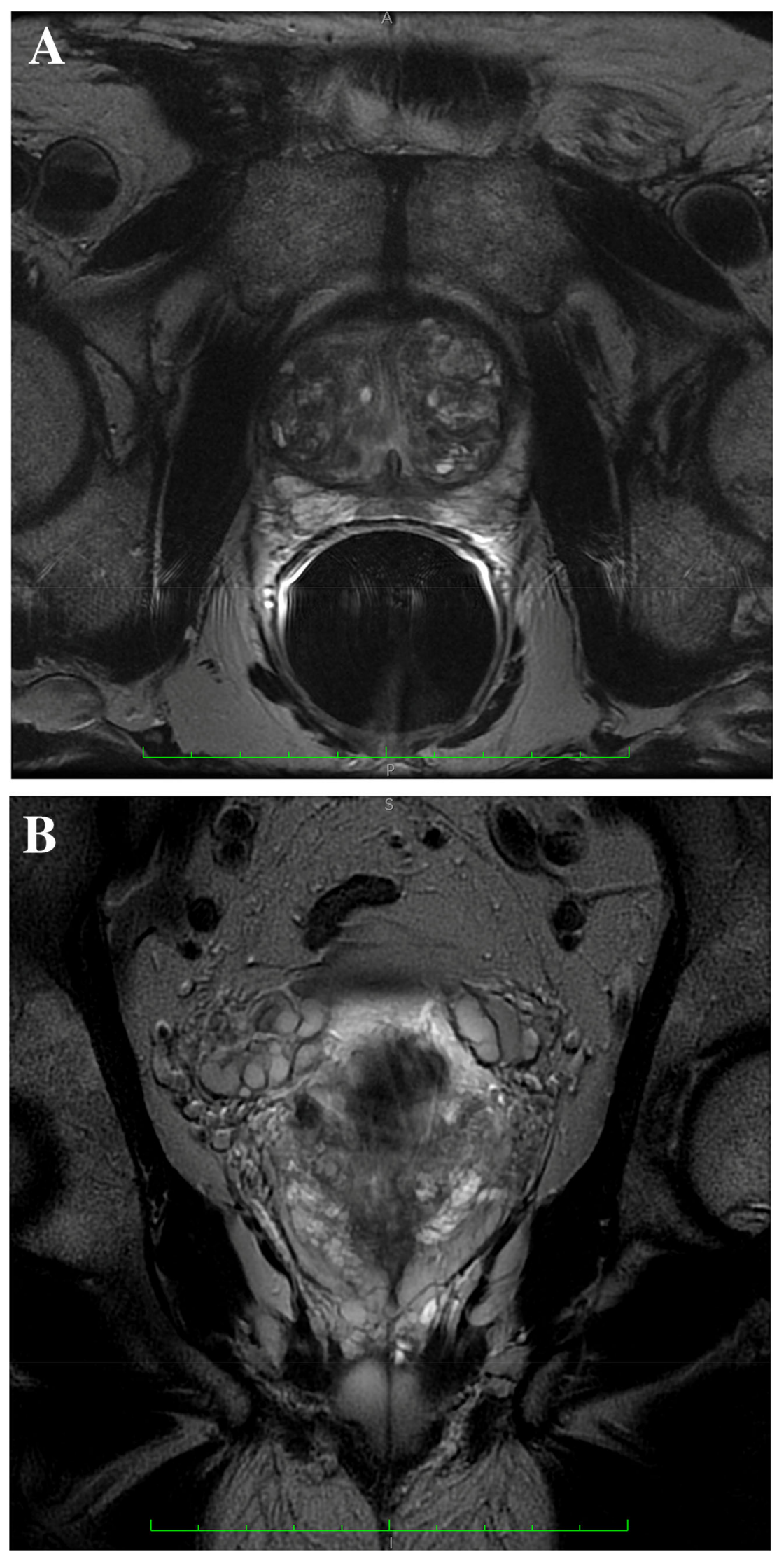


\section{Thick Surgical Capsule}

It is commonly called "surgical capsule" that band of fibromuscular and glandular tissue willing to separate the prostate TZ from the PZ. It is so called because of its surgical utility in constituting a landmark for the ablation or enucleation of nodules of benign prostatic hyperplasia (BPH) commonly affecting TZ [20, 21].

Although its band-like shape and its anatomical location are straights to recognize it, the surgical capsule may sometimes appear hypertrophic, especially in elderly patients, or even ill-defined if placed in close proximity to a prostatic carcinoma of TZ (Fig. 4).

For the above reasons, the assessment of anatomical sequences alone will not be sufficient to distinguish the surgical capsule from a possible prostate carcinoma, especially since both have a low signal in the T2WI sequences, and additionally, an equally low signal in the ADC map [9].

Unlike malignant neoplastic lesions, however, the surgical capsule shows no signal in DCE sequences; the latter given, in addition to anatomical characteristics, is therefore useful for carrying out a differential diagnosis [7•].

\section{Benign Conditions Mimicking Prostate Cancer at MR Imaging}

\section{Benign Prostatic Hyperplasia: Prostatic Stromal Nodule of BPH in Transition Zone (Tz) or Ectopic Nodule of BPH}

Benign prostatic hyperplasia (BPH) is a focal/nodular or diffuse growth of fibromuscular stromal and glandular tissue (each represented in variable percentage) of prostate which, in most cases, affects the TZ and which can cause a dimensional increase in the gland volume. This phenomenon shows a higher incidence from the age of 60 [22].

Due to the variable percentage of stromal and glandular tissue, a nodule of BPH may appear hypo/iso/hyper-intense in T2WI morphological sequences, thus showing the lower the signal intensity the higher the fibromuscular stromal content.

Usually the most frequent BPH nodules are those with a major stromal or mixed component and, for this reason, they appear as hypointense nodules in T2WI sequences in a manner almost identical to prostate cancer nodules [23]. In contrast, the greater representation of glandular tissue will give the nodule of BPH a hyperintense and sometimes cystic-like appearance in T2WI sequences, due to the greater fluid content of glandular secretions.

The evaluation of DWI/ADC map and DCE dynamic sequences also contributes significantly to making differential diagnosis even more complex. The nodules of $\mathrm{BPH}$, in fact, also show a low signal in the ADC map and a type 3 enhancement (early wash-in and rapid wash-out) after intravenous administration of contrast medium, similar characteristics to prostate cancer [24].

For these reasons, it is easy and quite frequent that a nodule of BPH with greater proliferation stromal can be mistaken for a carcinoma, then forcing the patient to undergo diagnostic deepening often invasive and uncomfortable, like the biopsy.

It is important to note, however, that prostate cancer affects PZ mainly and more frequently and that, on the other hand, TZ is more rarely affected [25].

Over the years, diagnostic strategies have been developed that can help the radiologist to provide a more accurate diagnosis, always supported by clinicalanamnestic data. Due to the overlapping behavior of the two types of lesions (benign/malignant) in DWI and dynamic sequences, it seems that the most significant sequences for differential diagnosis are the anatomical T2WI sequences that allow a morphological assessment of the suspected nodule [26].

The nodule of stromal hyperplasia (Fig. 5), usually has a round/oval shape, well defined margins and a thin surrounding pseudo-capsule $[6,26]$ characteristics that differentiate it from a possible carcinoma affecting the TZ, that has, instead, a more irregular and poorly defined morphology, an homogeneous low signal intensity at T2WI and usually tends to invade the anterior fibromuscular stroma (Fig. 6).

In case of doubt about differential diagnosis with T2WI sequences evaluation only, recent studies have demonstrated the validity of ADC sequences in distinguishing stromal glandular hyperplasia from prostatic carcinoma of $\mathrm{TZ}$, despite the many overlapping aspects.

Moreover, a quantitative assessment of signal intensity in the ADC map of suspected nodule (Fig. 7) may be a useful tool and in particular, it was found that lower ADC values correlate with a higher probability of malignant nodule $[27,28]$.

In some cases, it may happen that BPH nodules are found in the PZ of the prostate gland. This condition may be the result of two distinct events: either an ectopic nodule of hyperplasic TZ extending or herniating in the PZ (Fig. 8), and it can be recognized thanks to multiplanar prostate imaging, or more rarely that a nodule originating from the PZ itself and that, therefore, will be even more easily interpreted as a malignant lesion.

In conclusion, due to the characteristics of stromal BPH nodule comparable to those of prostatic carcinoma, the morphological and multiplanar study of the gland, but primarily of BPH nodule itself (its shape and its margins), 
Fig. 4 The arrows on ADC map (a) and axial T2WI (b) show, respectively, a focal area of low signal and hypointensity in right midgland near boundary of peripheral and transition zone. These findings are consistent with asymmetric thickening of surgical capsule, which appears on the controlateral side more typically thin and curvilinear
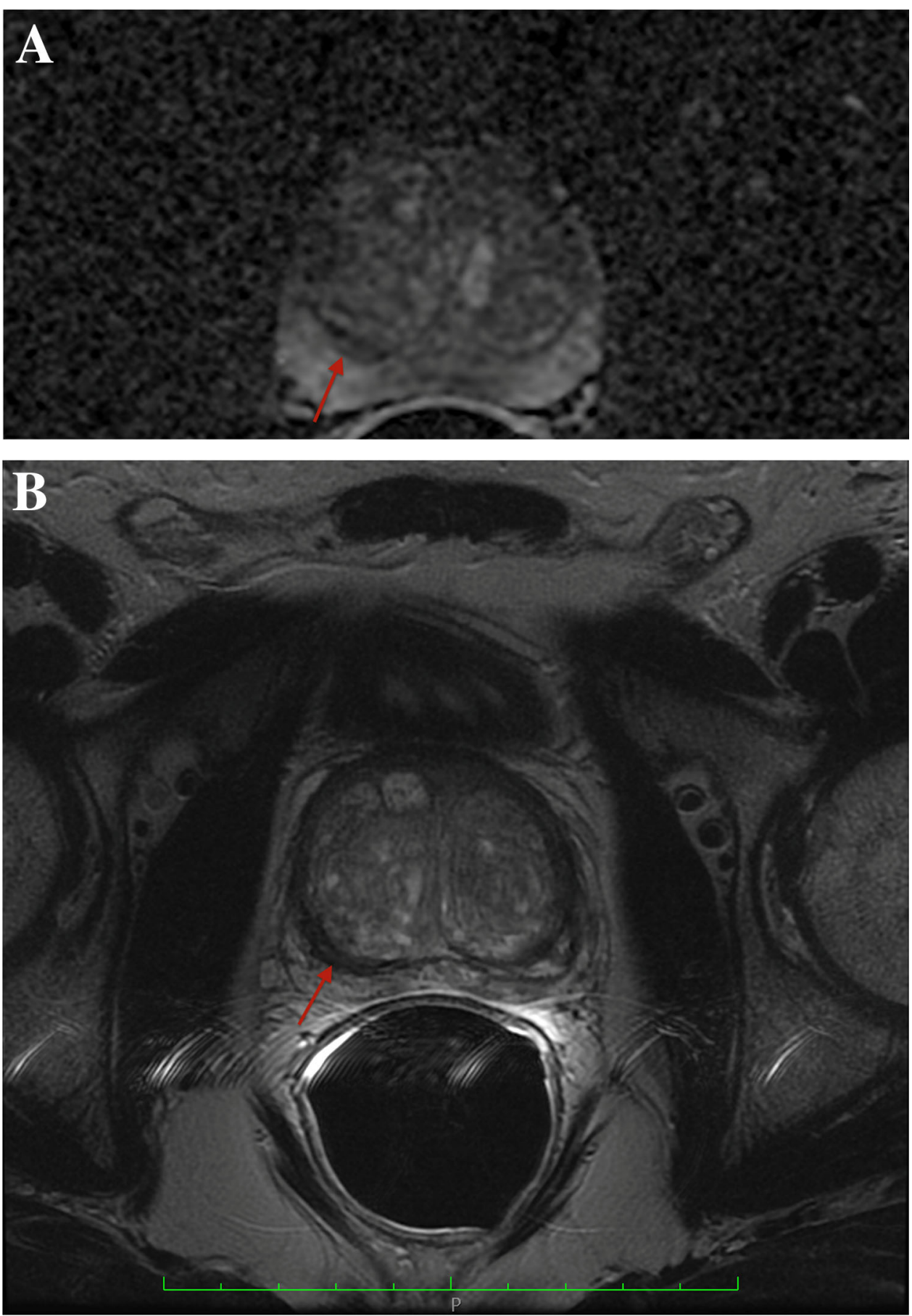

also considering its location in the TZ, are useful findings for differential diagnosis [29].

\section{Acute, Chronic and Granulomatous Prostatitis}

Prostatitis is a pathological condition with a bimodal age distribution, affecting the 20-40 age group and men over the age of 70 . It can be classified into numerous types and subtypes, starting with the two main groups: bacterial prostatitis and granulomatous prostatitis [1•].

Acute bacterial prostatitis, usually caused by E. coli or staphylococcus infections, manifests with a combination of local (genitourinary) and systemic symptoms and, if not properly treated, may evolve into chronic prostatitis.

In mp-MRI, focal prostatitis (Fig. 9), which usually affects PZ, appears as a nodular or linear area of reduced signal intensity in T2WI morphological sequences, shows 
Fig. 5 The arrows show a hypointense focal area on axial T2WI (a) of the right transition zone (TZ), which shows restricted diffusion on DWI (b) and ADC map (c). Looking in details at the T2WI axial sequence, it shows a round shape, well defined margins and a thin surrounding hypointense pseudo-capsule, characteristics that differentiate it from a possible carcinoma and more suggestive for a nodule of stromal hyperplasia
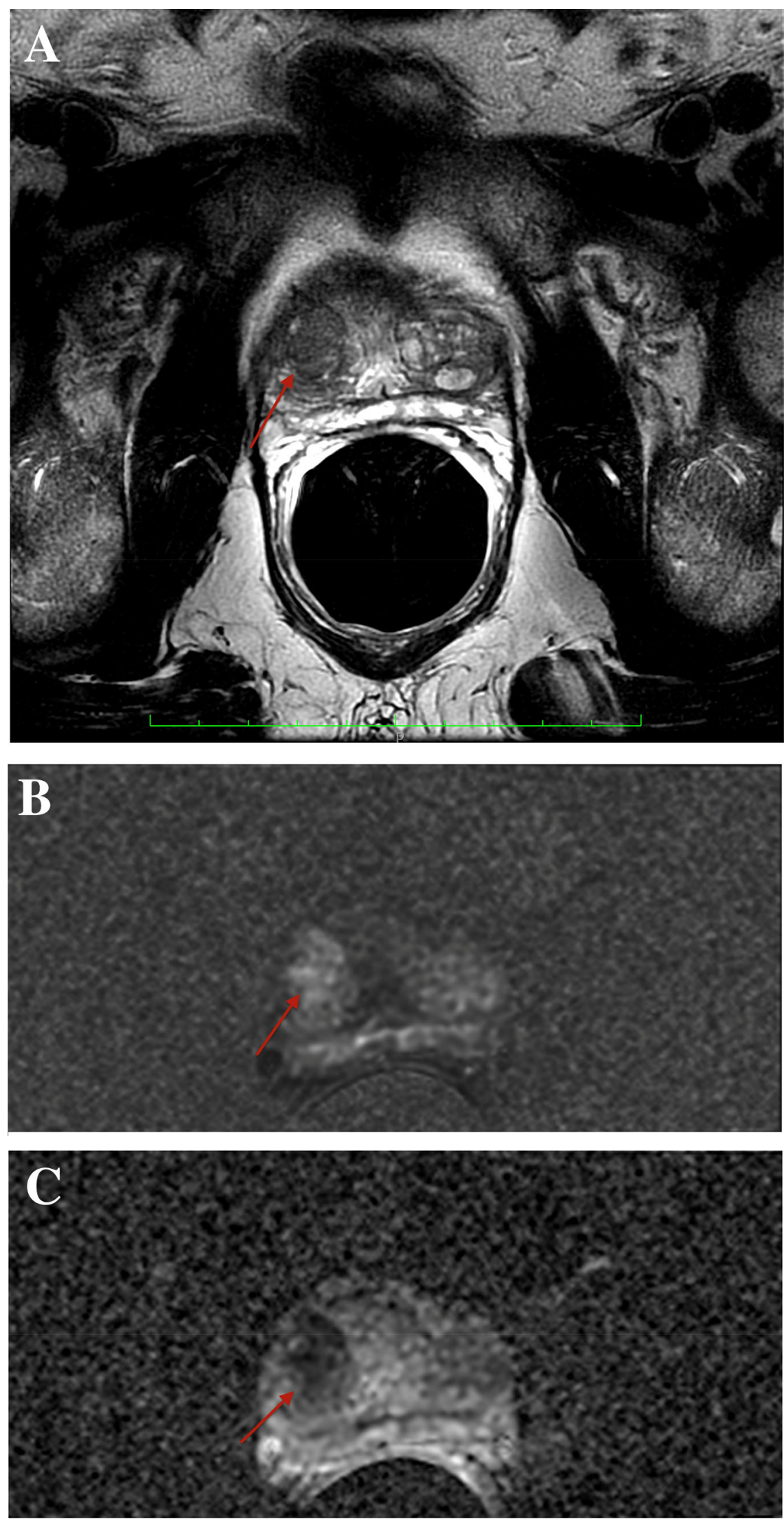
Fig. 6 The arrows show a hypointense focal area on axial T2WI (a) of the transition zone (TZ), which shows restricted diffusion on DWI (b) and ADC map (c). If compared with the nodular focal area of the Fig. 5, it has irregular and poorly defined morphology, a more homogeneous low signal intensity at T2WI and none surrounding pseudo-capsule. Suspicion of malignancy was confirmed by histological examination
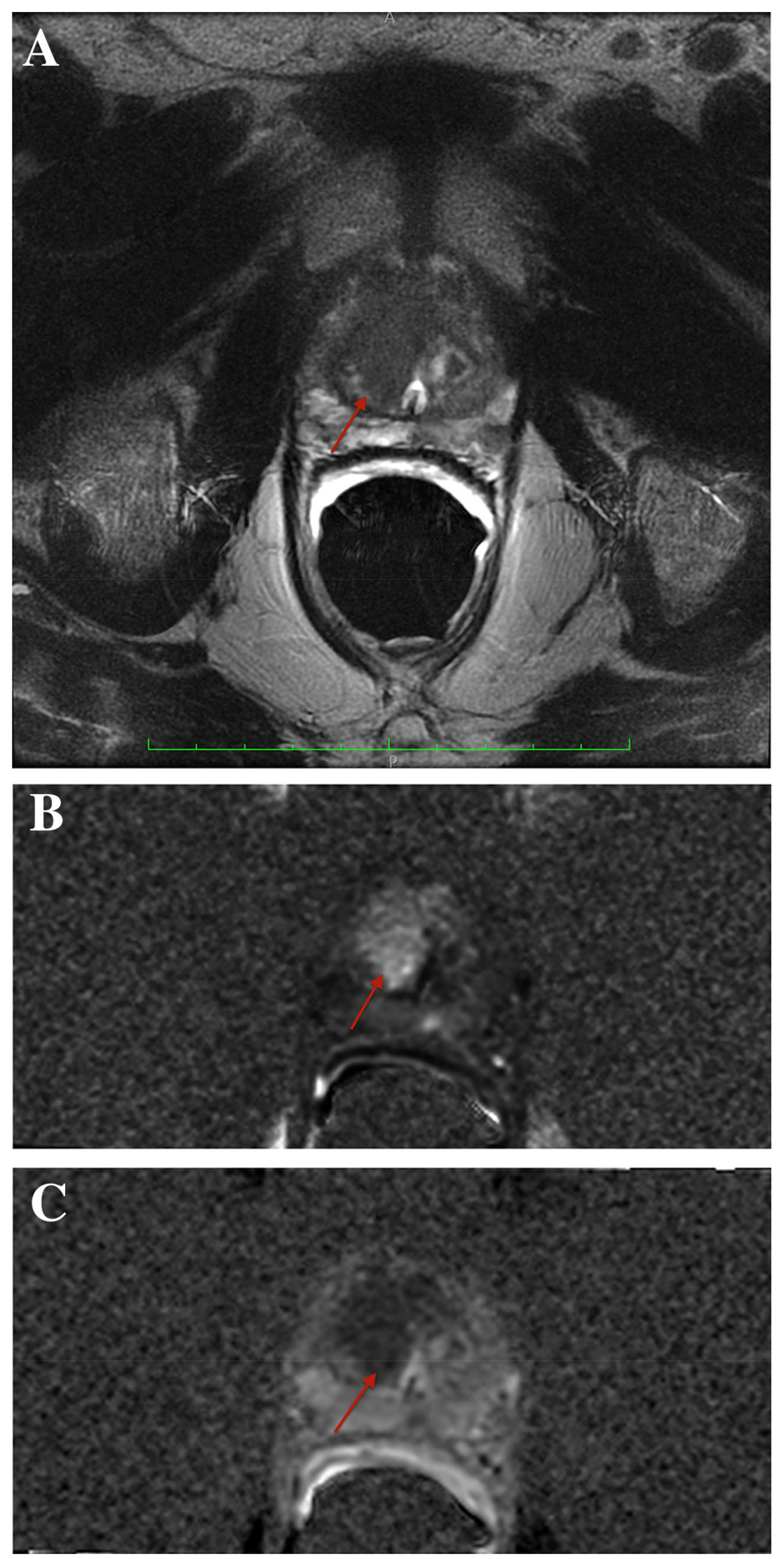
Fig. 7 Diffusely uneven

prostate gland with hypointense

irregular focal area $(20 \mathrm{~mm})$ on

axial T2WI (a) in the middle-

apical anterior right gland,

which shows restricted diffusion

on ADC map (b). Moreover, a

quantitative assessment of

signal intensity in the ADC map

results in a very low $\mathrm{ADC}$ value

(c), which correlates with a

higher probability of malignant

nodule. Suspicion of

malignancy was confirmed by

histological examination
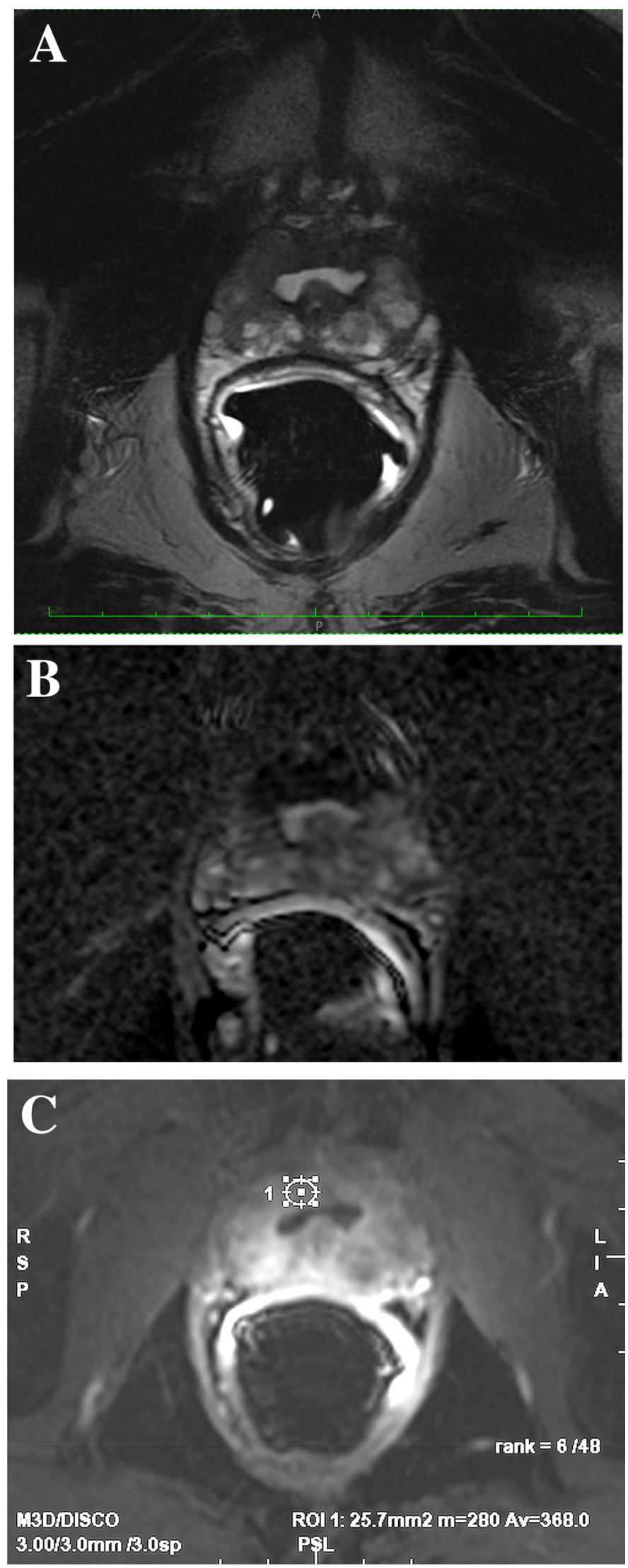

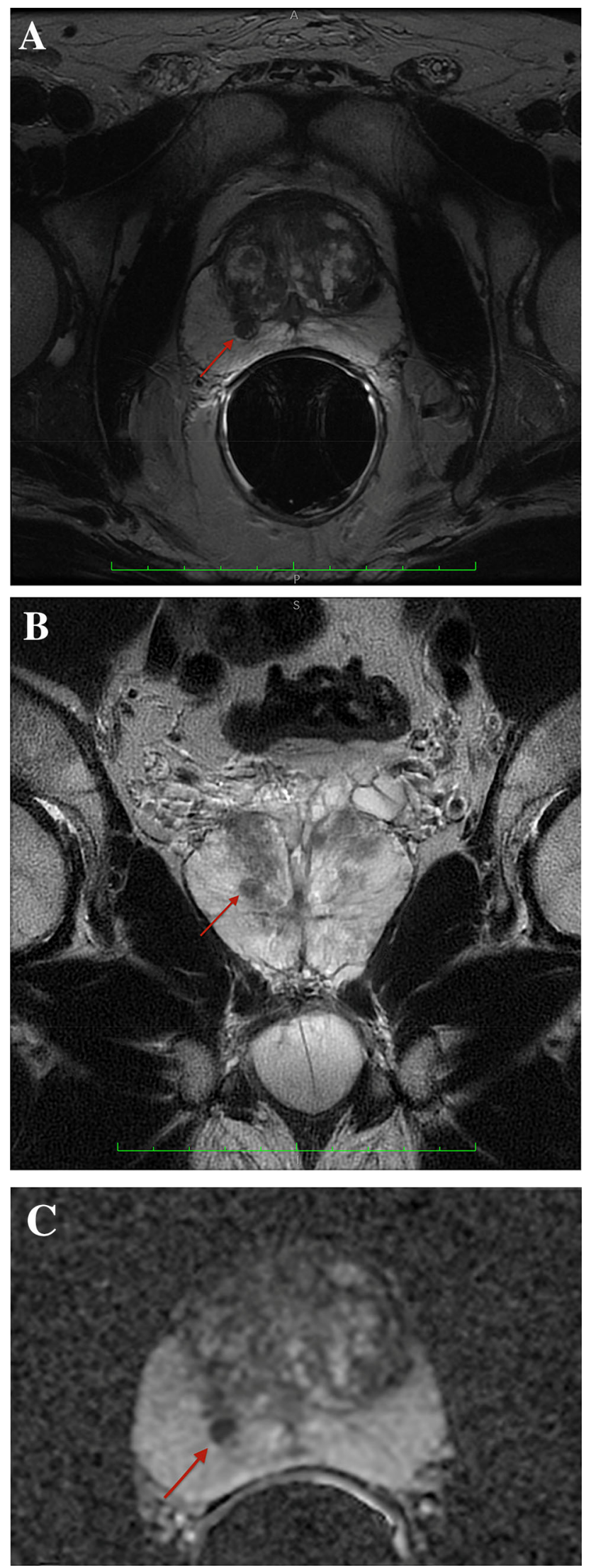

4Fig. 8 Focal nodule bulging in the right peripheral zone (arrows) which shows a low signal intensity on axial (a) and coronal (b) T2WI, with poorly defined margins and restrincted diffusion on the ADC map (c). It consists of a nodule of benign prostatic hyperplasia (BPH) from the central zone $(\mathrm{CZ})$ herniating in the peripheral zone $(\mathrm{PZ})$

restricted diffusion in DWI/ADC sequences, due to infiltration of inflammatory cells, and positive enhancement in DCE sequences [30, 31]. The described imaging characteristics are almost identical to those of prostate cancer, even more if we consider the common glandular localization (PZ) of both lesions (benign/malignant) and, in addition, the possibility of detecting an inflammatory infiltration in the periprostatic tissue, increased reactive locoregional lymph nodes and serum PSA levels above normal range, highly suggestive findings of clinically significant prostatic carcinoma [32].

Similar characteristics are also found in granulomatous prostatitis, a focal inflammatory condition of the prostate not frequently found and which, in addition to presenting itself as a common prostatitis, usually occurs with the formation of a hard-ligneous nodule at digital-rectal examination (DRE). It is in most cases idiopathic; other times, however, it may be the result of diagnostic-therapeutic procedures carried out directly on the prostate or on adjacent organs: such as a prostatic biopsy, a trans-urethral prostate resection (TURP) or intra-bladder instillation of the Calmette-Guerin bacillus (BCG) in the treatment of bladder cancer [33].

In the light of the above, it is clear that prostatitis is a real diagnostic challenge for the radiologist, since it has many aspects that can be compared to advanced prostate cancer. Unlike other conditions described above, in this case there is no more useful mp-MRI sequence than another; rather, correlation with clinical-anamnestic data and/or response to antibiotic therapy with lowering serum PSA levels are the most useful tools for appropriate diagnosis [34].

\section{Prostatic Haemorrhage}

It is very common that patients undergoing a diagnostic procedure in the suspicion of prostate cancer are subjected to biopsy, usually carried out by means of a trans-rectal ultrasound guided procedure at $\mathrm{PZ}$, and it is normal that it determines a minimal and localized bleeding rate.

The presence of blood, however, can last for a long time due to the physiological prostatic production of citrate, an endogenous anticoagulant that will therefore be responsible for the failure of blood clotting. In mp-MRI, particularly in the cardinal sequences of prostate imaging, blood takes on characteristics almost entirely similar to prostate cancer, 

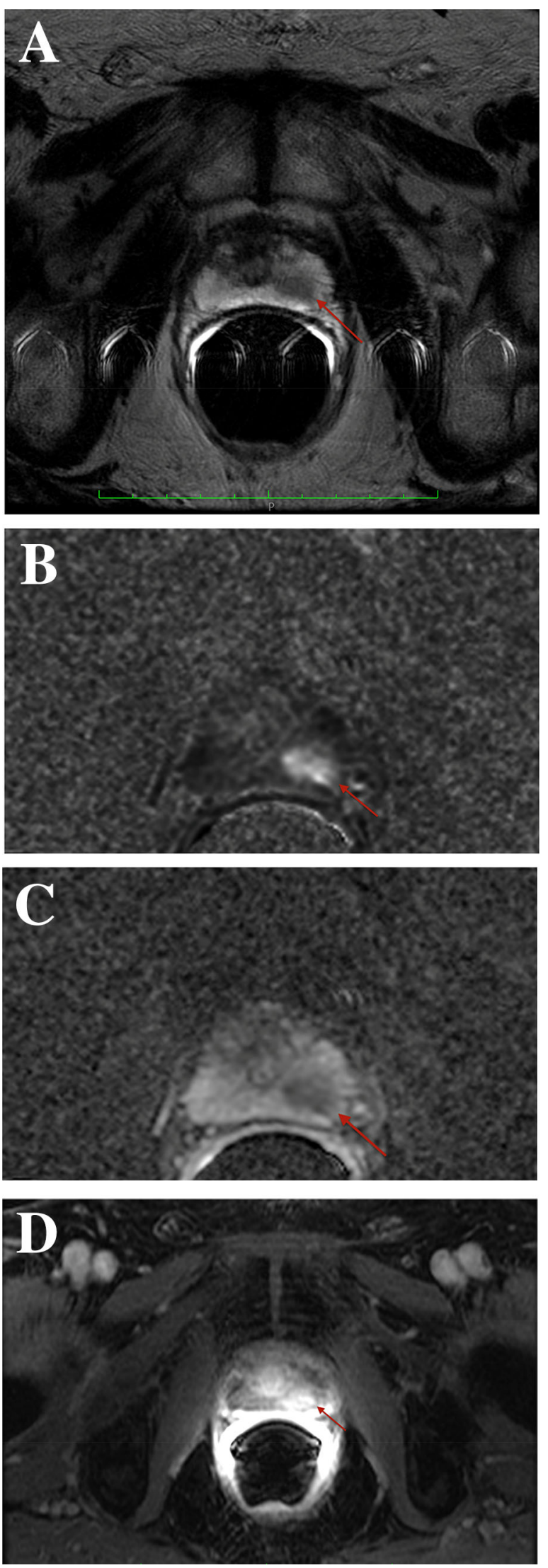

4Fig. 9 The arrows show a focal area of decreased signal in the left peripheral zone (PZ) on axial T2WI (a), a mild restriction on DWI (b) and ADC map (c) and a mild contrast uptake on DCE sequence (d). These findings are consistent with acute focal prostatitis

since it has a low signal in T2WI morphological sequences and, at times, especially in relation to the date of the haemorrhage, shows a restricted diffusion with hypointense signal in the ADC sequences [35]. Moreover, since the prostate cancer shows a high signal intensity on post-contrast T1WI, post-biopsy focus of haemorrhage may cover the malignant lesion and cause an overestimation of its extension. That's why, in these cases, it's almost imperative to add a T1WI-subtraction sequence in order to efface the haemorrhagic area, which would appear hyper-intense, and to let the prostate cancer be clearly visible [35].

It is, therefore, important for the radiologist to know how to recognize and distinguish a prostatic hemorrhage from a malignant lesion and, in order to do so, pre-contrast T1WI sequences are of primary importance because they allow us to see the haemorrhagic outbreak as a focal area with a high intensity signal, due to hemoglobin's degradation products [36].

In addition to the interpretation of imaging, it is important to keep in mind that post-biopsy haemorrhage regresses spontaneously and over time and, from numerous studies, it has emerged that the estimated average time for almost total resolution is about 6-8 weeks. Therefore, where possible, it is recommended to wait a proper latency time after the biopsy examination before subjecting the patient to a mp-MRI.

\section{Atrophy, Necrosis and Calcifications of Prostate}

Prostate atrophy is a very frequent histological finding, corresponding to about $73 \%$ of $\mathrm{PZ}$ biopsies [7•]. It is a consequence of a number of different pathogenic noxae that commonly affect the prostate, such as inflammation, radiant therapy, androgenic therapy and repeated ischemic insult.

In mp-MRI, prostatic atrophy occurs as a focal area of PZ with cuneiform morphology or geographic distribution across the prostate, resulting, therefore, a marked reduction in the volume of the gland [37].

It is important for the radiologist to know that prostatic atrophy is responsible for an increase in serum PSA values, likely due to a release of PSA into the blood by damaged prostatic epithelial cells, element that, in addition to imaging appearance, may be a confounding factor responsible for misdiagnosis and false positive results.

Atrophic prostatic tissue, in fact, shows a typically hypointense signal in T2WI sequences and a discreet signal 
Fig. 10 A focal area at the right PZ (arrow), adjacent to the coil, shows restricted diffusion on DWI (a). However, imaging T2WI sequence (b) do not show the same artifact, allowing us to exclude prostate cancer (it represents the typical appearance of mispositioned coil's artifact)
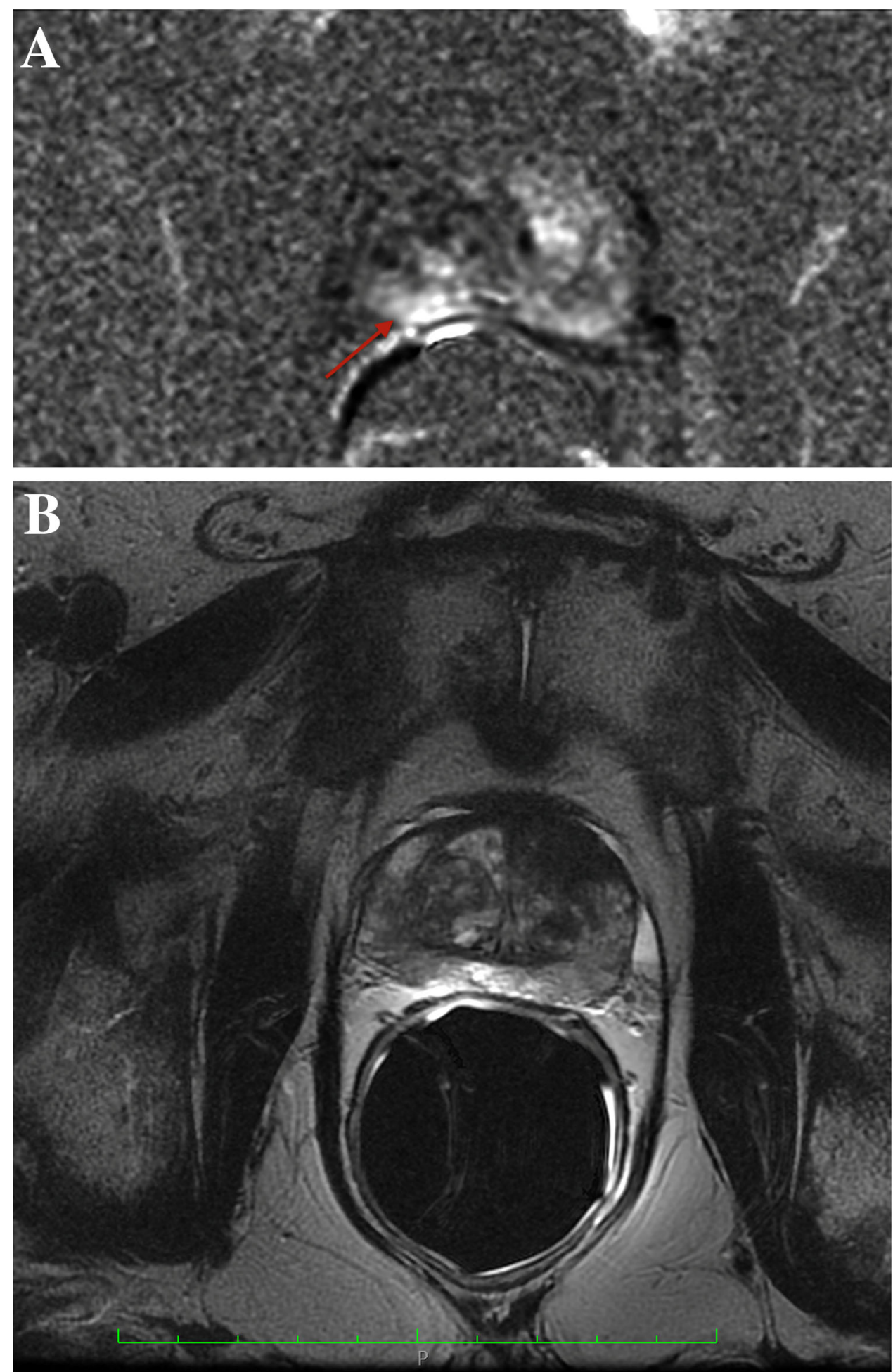

of restricted diffusion and enhancement that, however, will be less intense and less marked than prostate cancer [38].

This last aspect, together with an adequate clinicalanamnestic correlation, allows us to make a differential diagnosis.
The presence of necrotic areas of the prostatic gland is usually a consequence of infectious and/or inflammatory processes, as well as it is possible that they remain as a result of locoregional therapeutic procedures for the treatment of prostate cancer, as the cryoablation or laser or radiofrequency ablation. Specifically, from a histological 
point of view, these are well-defined areas of coagulative necrosis surrounded by areas of atrophy and inflammatory infiltrate.

In mp-MRI they show a markedly hypointense signal in T2WI sequences (much more than the hypo-intensity of prostatic carcinoma) and, due to the aforementioned histological characteristics, they show a restricted diffusion as evidence of the reduced water movement of necrotic tissue [4•].

The evaluation of dynamic sequences after intravenous administration of contrast medium, showing no evidence of enhancement, in addition to an adequate clinicalanamnestic correlation are suggestive findings of necrotic tissue.

Another frequent finding in prostate imaging, representing a potential diagnostic pitfall as well, is prostate calcifications, most commonly located at the junction between the $\mathrm{TZ}$ and the $\mathrm{PZ}$ and with an incidence progressively increasing with age. They are usually related to past pathological processes, such as BPH or prostatitis, and are due to the presence of concreted prostatic secretions or calcific corpora amylacea [39]. Due to the diamagnetic properties of calcium in mp-MRI, calcifications show a low signal in T2WI and ADC sequences, with an equally low signal intensity in DWI sequences at all b-values [7•], and do not exhibit enhancement in dynamic sequences.

\section{Technical Artifacts}

For the purpose of a correct differential diagnosis, in addition to the multiple pathological and para-physiologic conditions that can mimic prostate cancer, it is necessary to consider the possibility that there are also technical or patient-related pitfalls: peristaltic or patient-motion artifacts, image distortion, presence of rectal gas and/or feces, or endorectal coil malposition.

The use of an endorectal coil (ERC) is a topic still widely debated today, as it has not yet been established an absolute utility. Indeed, while the ERC, in addition to the surface one, improves spatial resolution and signal-to-noise ratio (SNR), due to its closeness to the prostate gland [5], it is responsible for artifacts and false images. For example, the presence of intestinal peristaltic movements creates concentric artifacts along the margins of the coil itself [23].

The correct positioning of the ERC is itself an important factor to obtain a high quality imaging; it should be positioned along a plane perpendicular to the left-right encoding direction, otherwise, a focal area of enhancement in the DCE sequences at the PZ, adjacent to the coil itself, and showing restricted diffusion on DWI is common (Fig. 10). However, imaging fees in ADC maps and T2WI sequences do not show the same artifact, allowing us to exclude prostate cancer [4•].

Since a large number of modern equipment, primarily $3 \mathrm{~T}$ but at the same time many $1.5 \mathrm{~T}$, are able to provide high quality and fairly satisfactory images from a diagnostic point of view, considering the above and, in addition, the discomfort created by the ERC, the PI-RADS committee does not recommend its use [5].

There is a broad spectrum of artifacts in mp-MRI, such as scaling, blurring, shading, cutting, and incorrect spatial recording, many of which are due to the presence of feces and gas in the rectal ampulla, due to high magnetic susceptibility. Rectal gas, for example, associated with intestinal peristaltic movements, may affect ADC calculation and DCE imaging [40].

To avoid the appearance of artifacts, it is possible to implement a series of measures prior to the execution of mp-MRI, such as the execution of an enema to empty the rectum from the stool or the administration of spasmolytic agents.

The latter aspect, however, is not free from risks and complications, since it should not be forgotten that many of the patients who undergo mp-MRI have urinary disorders often associated with BPH and, if so, the administration of spasmolytics can be highly damaging [4•].

\section{Conclusions}

Since its early use, mp-MRI has proved to be a very useful and increasingly widely used tool in the diagnosis and staging of prostate cancer, sometimes showing greater accuracy than canonical methodologies such as trans-rectal biopsy. This is also made possible by the existence of standardized protocols collected in PI-RADS v2.1, which are an indispensable guide for the radiologist in the evaluation of mp-MRI of the prostate, highlighting the main sequences used in the detection of prostatic cancer also in relation to the zonal anatomy of the gland.

Despite this, there are numerous pathological and paraphysiological conditions or anatomical structures of the prostate gland that mimic prostate carcinoma both for morphological characteristics and intraglandular localization (especially those located in the PZ), and for their appearance in anatomical (T2WI) and functional (DWI/ ADC and DCE) mp-MRI.

Therefore, it will be important for the radiologist not only the accurate study of prostate imaging on axial, sagittal and coronal planes and in all sequences, but also knowledge of the main pitfalls listed here to avoid diagnostic errors caused by a misinterpretation of the images. 
Acknowledgements Special thanks are given to Fabio Carbonari, RT at the Radiology Unit "Carlo Urbani" Hospital, for the excellent work in carrying out the mp-MRI examinations.

Funding Open Access funding provided by Università di Foggia.

\section{Compliance with Ethical Standards}

Conflict of interest Each Author declares that they have no commercial associations (e.g. consultancies, stock ownership, equity interest, patent/licensing arrangement etc.) that might pose a conflict of interest in connection with submitted article.

Open Access This article is licensed under a Creative Commons Attribution 4.0 International License, which permits use, sharing, adaptation, distribution and reproduction in any medium or format, as long as you give appropriate credit to the original author(s) and the source, provide a link to the Creative Commons licence, and indicate if changes were made. The images or other third party material in this article are included in the article's Creative Commons licence, unless indicated otherwise in a credit line to the material. If material is not included in the article's Creative Commons licence and your intended use is not permitted by statutory regulation or exceeds the permitted use, you will need to obtain permission directly from the copyright holder. To view a copy of this licence, visit http://creativecommons. org/licenses/by/4.0/.

\section{References}

Recently published papers of particular interest have been highlighted as:

- Of important because it contains all the most common pitfalls of radiologist's daily practice,showing the main features and illustrating the possible strategies to put in place to avoid them.

- Of major importance because it refers to the last recommendation ofPI-RADS correlating theme with the most common pitfalls in prostatic mp-MRI

1. - Catalá V, et al. "Multiparametric MRI and prostate cancer: pitfalls and tricks. In: Atlas of multiparametric prostate MRI. Cham: Springer; 2018. p. 77-113.

2. Ghai S, Haider M. Multiparametric-MRI in diagnosis of prostate cancer. Indian J Urol. 2015;31(3):194.

3. Weinreb JC, et al. PI-RADS prostate imaging-reporting and data system: 2015, version 2. EurUrol. 2016;69:16-40.

4. - Panebianco V, Giganti F, Kitzing YX, et al. An update of pitfalls in prostate mpMRI: a practical approach through the lens of PI-RADS v. 2 guidelines. Insights Imaging. 2018;9(1):87-101.

5. Ventrella E, Eusebi L, Carpagnano FA, et al. Multiparametric MRI of prostate cancer: recent advances. CurrRadiol Rep. 2020;8:19.

6. Panebianco V, et al. Pitfalls in interpreting mp-MRI of the prostate: a pictorial review with pathologic correlation. Insights Into Imaging. 2015;6(6):611-30.

7. - Chatterjee A, Stephen T, Aytekin O. Prostate MR: pitfalls and benign lesions. AbdomRadiol. 2019. https://doi.org/10.1007/ s00261-019-02302-x.

8. Niaf E, Lartizien C, Bratan F, et al. Prostate focal peripheral zone lesions: characterization at multiparametric MR imaging: influence of a computer-aided diagnosis system. Radiology. 2014;271(3):761-9.

9. Kitzing YX, et al. Benign conditions that mimic prostate carcinoma: MR imaging features with histopathologic correlation. Radiographics. 2016;36(1):162-75.

10. McNeal JE, et al. Normal and pathologic anatomy of prostate. Urology. 1981;17(suppl 3):11-6.

11. Allen KS, Kressel HY, Arger PH, Pollack HM. Age-related changes of the prostate: evaluation by MR imaging. AJR Am J Roentgenol. 1989;152(1):77-81.

12. Ward E, Baad M, Peng Y, et al. Multi-parametric MR imaging of the anterior fibromuscularstroma and its differentiation from prostate cancer. AbdomRadiol (NY). 2017;42(3):926-34.

13. Cristini C, Di Pierro GB, Leonardo C, De Nunzio C, Franco G. Safe digital isolation of the santorini plexus during radical retropubic prostatectomy. BMC Urol. 2013;13:13.

14. Caglic I, Viljem K, Barrett T. Multiparametric MRI-local staging of prostate cancer and beyond. RadiolOncol. 2019;53(2):159-70.

15. Rosenkrantz AB. Radiologist, be aware: ten pitfalls that confound the interpretation of multiparametric prostate MRI. Am J Roentgenol. 2014;202:109-20.

16. Vargas HA, Akin O, Franiel T, et al. Normal central zone of the prostate and central zone involvement by prostate cancer: clinical and MR imaging implications. Radiology. 2012;262(3):894-902.

17 Cohen RJ, Shannon BA, Phillips M, Moorin RE, Wheeler TM, Garrett KL. Central zone carcinoma of the prostate gland: a distinct tumor type with poor prognostic features. J Urol. 2008;179(5):1762-7 (discussion 7).

18. Gupta RT, Kauffman CR, Garcia-Reyes K, et al. Apparent diffusion coefficient values of the benign central zone of the prostate: comparison with low- and high-grade prostate cancer. AJR Am J Roentgenol. 2015;205(2):331-6.

19. Hansford BG, Karademir I, Peng Y, et al. Dynamic contrastenhanced MR imaging features of the normal central zone of the prostate. AcadRadiol. 2014;21(5):569-77.

20. Semple JE. Surgical capsule of the benign enlargement of the prostate: its development and action. BMJ. 1963;1(5346):1640-3.

21. Kahokehr AA, Gilling PJ. Which laser works best for benign prostatic hyperplasia? CurrUrol Rep. 2013;14(6):614-9.

22. Guneyli S, Ward E, Thomas S, et al. Magnetic resonance imaging of benign prostatic hyperplasia. DiagnIntervRadiol. 2016;22(3):215-9.

23. Heijmink SW, Fütterer JJ, Hambrock T, et al. Prostate cancer: body-array versus endorectal coil MR imaging at $3 \mathrm{~T}$ - comparison of image quality, localization and staging performance. Radiology. 2007;244(1):184-95.

24. Shannon BA, McNeal JE, Cohen RJ. Transition zone carcinoma of the prostate gland: a common indolent tumor type that occasionally manifests aggressive behavior. Pathology. 2003;35(6):467-71.

25. Hoeks CM, Hambrock T, Yakar D, de Kaa CAH, Feuth T, Witjes JA, et al. Transition zone prostate cancer: detection and localization with 3-T multiparametric MR imaging. Radiology. 2013;266(1):207-17.

26. Kayhan A, Fan X, Oommen J, Oto A. Multi-parametric MR imaging of transition zone prostate cancer: imaging features, detection and staging. World J Radiol. 2010;2(5):180-7.

27. Oto A, Kayhan A, Jiang Y, et al. Prostate cancer: differentiation of central gland cancer from benign prostatic hyperplasia by using diffusion-weighted and dynamic contrast-enhanced MR imaging. Radiology. 2010;257(3):715-23.

28. Verma S, Turkbey B, Muradyan N, et al. Overview of dynamic contrast-enhanced MRI in prostate cancer diagnosis and management. AJR Am J Roentgenol. 2012;198:1277-88. 
29. Tang J, Yang JC, Zhang Y, et al. Does benign prostatic hyperplasia originate from the peripheral zone of the prostate? A preliminary study. BJU Int. 2007;100(5):1091-6.

30. Coker TJ, Dierfeldt DM. Acute bacterial prostatitis: diagnosis and management. Am Fam Physician. 2016;93(2):114-20.

31. Holt JD, Garrett WA, McCurry TK, Teichman JM. Common questions about chronic prostatitis. Am Fam Physician. 2016;93(4):290-6.

32. Meier-Schroers M, Kukuk G, Wolter K, et al. Differentiation of prostatitis and prostate cancer using the Prostate Imaging-Reporting and Data System (PI-RADS). Eur J Radiol. 2016;85(7):1304-11.

33 Rais-Bahrami S, Nix JW, Turkbey B, et al. Clinical and multiparametric MRI signatures of granulomatous prostatitis. AbdomRadiol (NY). 2017. https://doi.org/10.1007/s00261-0171080-0.

34. Ramakrishnan K, Salinas R. Prostatitis: acute and chronic. Prim Care. 2010;37(3):547-63.

35. Rosenkrantz AB, Kopec M, Kong X, et al. Prostate cancer vs post-biopsy hemorrhage: diagnosis with T2- and diffusionweighted imaging. J MagnReson Imaging. 2010;31(6):1387-94.
36. Sharif-Afshar AR, Feng T, Koopman S, et al. Impact of post prostate biopsy hemorrhage on multiparametric magnetic resonance imaging. Can J Urol. 2015;22(2):7698-702.

37. Benedetti I, Bettin A, Reyes N. Inflammation and focal atrophy in prostate needle biopsy cores and association to prostatic adenocarcinoma. Ann DiagnPathol. 2016;24:55-61.

38. Freitas DM, Andriole GL Jr, Castro-Santamaria R, Freedland SJ, Moreira DM. Extent of baseline prostate atrophy is associated with lower incidence of low- and high-grade prostate cancer on repeat biopsy. Urology. 2017;103:161-6.

39. Sfanos KS, Wilson BA, De Marzo AM, Isaacs WB. Acute inflammatory proteins constitute the organic matrix of prostatic corpora amylacea and calculi in men with prostate cancer. ProcNatlAcadSci USA. 2009;106(9):3443-8.

40. Caglic I, Hansen NL, Slough RA, Patterson AJ, Barrett T. Evaluating the effect of rectal distension on prostate multiparametric MRI image quality. Eur J Radiol. 2017;90:174-80.

Publisher's Note Springer Nature remains neutral with regard to jurisdictional claims in published maps and institutional affiliations. 